\section{Prospective study of asthma in relation to smoking habits}

SIR,-Dr E Vesterinen and colleagues (July 1988;43:534-9) reported an increase in relative risk of asthma with smoking, consistent with suggestions that smoking promotes the inception of asthma and its relapse,' though the increase was only small. These data should be considered in the context of other studies suggesting a stronger risk.

There is consistent evidence, which we have summarised in part, ${ }^{23}$ that tobacco smoke (and other irritants, such as ozone) has a substantial adjuvant effect on IgE formation, and hence IgE mediated asthma. It is based on studies of adults exposed to occupational allergens and of infants (in relation to maternal smoking), and on experiments in animals. The proportion of asthma which is IgE mediated is unknown, but this does not diminish interest in causal mechanisms for its inception, in the same way that carcinogenesis by smoke is of general interest even though it is specific to certain cancers.

This effect may be detectable in the general population but in the study of Vesterinen and colleagues could be balanced by other factors. The study was large and could not be expected to distinguish IgE mediated from other asthma. More importantly, the design did not separate the effect of smoking on asthma from the influence of asthma on smoking habit. The difficulty in distinguishing causes from consequences of illness is a major argument against drawing causal inferences from prevalence data. An unknown, perhaps high, proportion of patients in the prevalence analysis would have had asthma from childhood, as would some of the incident cases. The data showed that adults with asthma are more likely to stop smoking and to remain non-smokers than others and it is probable that adolescents with a history of asthma are less likely to take up smoking than others.

Although Dr Vesterinen and colleagues used the best indices of asthma available to them, these probably added further bias. One was a self reported history of asthma diagnosis, which may be made preferentially in non-smokers. ${ }^{1}$ The second was admission or death coded by ICD 8, which excludes asthma when associated with bronchitis or emphysema, thus preferentially excluding smokers with asthma. A future analysis using ICD 9 would be of interest.

It is likely that a positive relation with smoking at one or more of the several steps leading to asthma in adults was balanced by competing negative bias, making the study uninformative aetiologically. It, has however, public health value: a small increase in relative risk when both exposure and disease are common, as in passive smoking and lung cancer, implies that a large number of cases could be prevented by reducing exposure. This is also the case for smoking and asthma and this study has added one more argument in favour of limiting smoking.

KM VENABLES AJ NEWMAN TAYLOR Department of Occupational Medicine National Heart and Lung Institute Brompton Hospital London

1 Burrows B. The natural history of asthma. J Allergy Clin Immunol 1987;80:373-7.
2 Venables KM. Epidemiology and the prevention of occupational asthma. Br J Ind Med 1987;44:73-5.

3 Newman Taylor AJ. Allergic lung disease. In: Dawson AM, Besser GM, eds. Recent advances in medicine 20. Edinburgh: Churchill Livingstone, 1987:143-60.

Does right ventricular function predict survival in patients with chronic obstructive lung disease?

SIR,-I read with great interest the article of Dr AJ France and colleagues (August 1988;43:621-6) concerning the effect of right ventricular function on survival in patients with chronic obstructive lung disease.

In the article, there is no information about the patient's medication. Had the patients taken any medicament (digitalis, nitrates, diuretics, calcium channel blockers, converting enzyme inhibitors, etc) before the study that might improve cardiac pump function or reduce pulmonary vasculature resistance? If so, were the drugs stopped before radionuclide ventriculography?

A second question is whether any of the 115 patients had atrial fibrillation. How reliable is the evaluation of ejection fraction with radionuclide ventriculography in patients with atrial fibrillation?

MAHMUT KARAKUZU
Department of Internal Medicine
Dokuz Eylül University
Izmir
Turkey

AUTHOR'S REPLY I would like to thank Dr Karakuzu for his interest in our paper. In answer to his questions the drug therapy of our patients consisted of inhaled bronchodilators (beta $a_{2}$ agonists and/or ipratropium) in all patients and inhaled steroids in a few. Those patients with oedema at the time of study or in the past were also taking varying doses of diuretics. None of the patients was taking any of the drugs he mentions which may improve cardiac "pump" function, and this also included oral theophyllines. We do not believe that there is any evidence to suggest that chronic diuretic therapy improves cardiac "pump" function.

We did not study any patient with atrial fibrillation. The movie images obtained by gated equilibrium radionuclide ventriculography in patients with uncontrolled atrial fibrillation are often of poor quality, making it difficult to outline the ventricles accurately, thus reducing the accuracy of the ejection fraction obtained. The image can be improved by setting limits for the $\mathbf{R}-\mathbf{R}$ interval and acquiring data over a greater number of cardiac cycles or indeed by controlling the ventricular rate with digoxin. We also chose not to study patients with atrial fibrillation since this might indicate underlying occult ischaemic heart disease.

W MACNEE

Department of Respiratory Medicine City Hospital Edinburgh 